\title{
Protecting the patient is our protection
}

\author{
Simonetta Piana ${ }^{1}$, Zoe Apalla ${ }^{2}$
}

\author{
1 Pathology Unit, IRCCS-Arcispedale Santa Maria Nuova, Reggio Emilia, Italy \\ 2 First Department of Dermatology, Medical School, Aristotle University of Thessaloniki, Greece
}

\begin{abstract}
Citation: Piana S, Apalla Z. Protecting the patient is our protection. Dermatol Pract Concept. 2014;4(1):1. http://dx.doi.org/10.5826/ dpc.0401a01

Copyright: (92014 Piana et al. This is an open-access article distributed under the terms of the Creative Commons Attribution License, which permits unrestricted use, distribution, and reproduction in any medium, provided the original author and source are credited.

Corresponding author: Simonetta Piana, Pathology Unit, IRCCS-Arcispedale Santa Maria Nuova, Reggio Emilia, Italy. Email: simonetta. piana@asmn.re.it.
\end{abstract}

Optimization and securing of medical practices and procedures has always been very challenging in medicine. However, the real-life clinical settings continue to pose various hazards that may lead medical professionals to unintended mistakes.

Pathologists feel particularly responsible and are very concerned about diagnostic discrepancies or even mistakes, since these depend on their interpretive skills and diagnostic expertise. However, a majority of mistakes in daily practice are related to laboratory procedural errors, involving transportation of the specimens, labeling, contamination, cutting, typing, and various others [1]. In contrast to general belief, errors in diagnostic interpretation account for only $25 \%$ of the overall failures in surgical pathology practice, with most of the remaining $75 \%$ resulting from defective specimens, incorrect patient identification, mislabeling and/or inadequate reports [2].

Dermatopathology is considered to be the pathology subspecialty associated with higher medico-legal risk [3]. It is well known that the most serious problems in dermatopathology emanate from the incorrect interpretation of melanocytic lesions. However, diagnostic limitations are not the only weakness in dermatopathology practice.

Although underestimated, the handling of large numbers of small, similar-looking bioptic specimens results very often in unpredictable mistakes. The latter risk is scarcely reported in the literature and has rarely been investigated, since its objective quantification is not easy. There are many vulnerable steps in the routine of a pathology lab, from the initial access to the lab until the final report and communication with the patient's physician. Errors pervade all levels of the pre-analytical, analytical, and post-analytical phases, especially in labs that accept a high volume of specimens [3]. The pre-analytical phase, from the arrival at the lab to the commencement of the analysis, is considered the most vulnerable to faults [4]. Receiving the specimen, incorporating the tissue in paraffin blocks and tissue cutting are procedures susceptible to random mistakes and should be systematically controlled [4]. It is generally accepted that the risk of mistakes increases with the number of samples and with the complexity of the required procedure to establish a definite diagnosis. The routine use of immunohistochemical techniques and the increasing interest in new biomolecular markers, which influence management decisions, complicate further this already complex scenario.

The article by Weyers included in this issue analyzes the step-by-step process carried out in a pathology lab, outlining the handling of samples and the risk of specimen mix-up. According to the author, a dermatopathology lab could be likened to a factory production line, as it continuously processes and reports on many skin specimens; and in this context, lab mechanisms are particularly susceptible to faults. The author describes all the pathways a biopsy routinely takes, highlighting the pitfalls and recommending preventive strategies for every stage of the procedure. In the era of targeted therapies, when a molecular biology test can be therapeutically strategic, it is crucial to ensure the optimal quality of tissue samples by securing every diagnostic step.

Addressing pathology errors and eliminating potential sources of mistakes go hand-in-hand with the right of the patients and their caregivers to a complete, correct, and timely diagnosis. Apart from the aforementioned issues, protecting the patient is also achieved by the accurate and effectivequality checks throughout the system, which in turn protects the physician against malpractice claims while working in the difficult, sometimes under-recognized, but always wonderful, job of dermatopathology. 


\section{References}

1. Sirota RL. Defining error in anatomic pathology. Arch Pathol Lab Med. 2006;130(5):604-6.

2. Meier FA, Varney R, Bansal M, et al. Validation of an error taxonomy system for anatomic pathology (abstract). Mod Pathol. 2005;18:1506.
3. Troxel DB. Medicolegal aspects of error in pathology. Arch Pathol Lab Med. 2006;130(5):617-9.

4. Novis DA. Detecting and preventing the occurrence of errors in the practices of laboratory medicine and anatomic pathology: 15 years' experience with the College of American Pathologists' Q-PROBES and Q-TRACKS programs. Clin Lab Med. 2004;24(4):965-78. 